\title{
AUTONOMIA E O USO DE RECURSOS DIGITAIS NO ENSINO-APRENDIZAGEM DE INGLÊS
}

RESUMO: O presente artigo, organizado em duas partes, busca oferecer subsídios teóricos e práticos ao focalizar o uso de recursos digitais no desenvolvimento da autonomia dos agentes envolvidos no processo de ensino-aprendizagem de inglês. Na primeira parte, de natureza teórica, orientados pela teoria da complexidade, propomos oferecer uma breve visão do conceito de autonomia como sistema adaptativo complexo. Na segunda parte, de natureza prática, buscamos apresentar possíveis usos de diversos recursos digitais, disponíveis gratuitamente na internet, que possam contribuir positivamente para a promoção da autonomia de professores e aprendizes.

PALAVRAS-CHAVE: recursos digitais; ensino de inglês; autonomia; teoria da complexidade

\section{Introdução}

O termo "autonomia" é frequentemente associado ao aprendiz, e a expressão autonomia discente (do inglês learner autonomy), estabelecida por Carl Rogers em 1969, refere-se à habilidade, que todos os aprendizes possuem, em diferentes níveis, de desenvolver um planejamento de estudo pessoal, de encontrar materiais para pesquisar e de avaliar, por si mesmos, o progresso (ou não) de sua aprendizagem. Rogers afirma que um dos princípios para a aprendizagem, fundamental para o conceito de autonomia, é a liberdade de escolha. Podemos entender, portanto, que professores e alunos autônomos são aqueles indivíduos livres para decidir a maneira mais adequada de atingir seus objetivos de ensino e de aprendizagem, respectivamente.

Inseridos em contextos mediados por tecnologias digitais, docentes e alunos encontram inúmeras possibilidades para desenvolver sua autonomia. Segundo Tavares e Franco (2014), a internet é tanto fonte e veículo de distribuição de informação quanto meio de

\footnotetext{
* Doutor em Estudos Linguísticos pela Universidade Federal de Minas Gerais. Professor Associado do Setor de Inglês do Departamento de Letras Anglo-Germânicas da Universidade Federal do Rio de Janeiro (UFRJ) Pesquisador do Projeto LingNet (UFRJ).
} 
comunicação entre pessoas. Com base nessas duas características da internet, argumentamos, ao longo deste artigo, que ela pode propiciar o desenvolvimento da autonomia de professores e aprendizes.

\section{Autonomia como sistema complexo}

Emprega-se o termo autonomia, geralmente, associado às ideias de independência, liberdade, controle, monitoramento, gerenciamento, responsabilidade, entre outras. Ao mesmo tempo, compreende-se autonomia como algo que se distancia de autoinstrução, isto é, de aprender sem a ajuda do professor. Em estudo realizado em 2006, Paiva revê alguns conceitos de autonomia na área de aquisição de segunda língua e conclui que o termo é bem mais complexo ${ }^{1}$ do que possamos imaginar.

Para alguns autores (HOLEC, 1981; DICKINSON, 1991; LITTLE, 1991), a autonomia está relacionada, fundamentalmente, à responsabilidade que o aprendiz possui sobre sua própria aprendizagem, sem considerar fatores externos. Dessa forma, a partir do momento que o indivíduo passa a ter controle da própria aprendizagem, buscando fontes que o ajudem a desenvolver seu potencial, ele adquire graus de autonomia.

Entretanto, como Paiva (2006) argumenta, estudar sozinho não representa necessariamente ter uma atitude autônoma, pois as decisões tomadas e o caminho percorrido são, na maioria das vezes, determinados pelo material didático.

Para outros autores (FREIRE, 2007; PENNYCOOK, 1997; BENSON, 1987), a autonomia parece despertar, no aprendiz, o sentimento de liberdade. Freire (2007), por exemplo, afirma que a autonomia deve ser entendida como um direito para a própria produção ou construção de conhecimento. Segundo o educador, "o respeito à autonomia e à dignidade de cada um é um imperativo ético e não um favor que podemos ou não conceder uns aos outros" (2007, p. 59).

A autonomia, na visão de Paiva, é essencialmente parte da aquisição de uma segunda língua, e esses dois sistemas são complexos. Uma característica essencial do sistema complexo ou dinâmico é a auto organização. Para a pesquisadora, “a autonomia do aprendiz pode auto organizar a aquisição, pois os processos cognitivos e algumas escolhas de aprendizagem dependem dos aprendizes" (2006, p. 92).

Outras características de um sistema complexo, conforme aponta Larsen-Freeman (1997), são: dinamicidade, não linearidade, caos, imprevisibilidade, sensibilidade às condições

\footnotetext{
${ }^{1} \mathrm{O}$ emprego do vocábulo complexo se distancia da noção de algo complicado, sugerido pelo uso comum da palavra: de acordo com a teoria da complexidade, trata-se de um fenômeno que compreende inúmeras possibilidades de interações e indeterminações.
} 
iniciais e ao feedback, abertura e adaptabilidade. Ao retomar algumas características dos sistemas complexos, Franco (2013) destaca que: esses sistemas são formados por elementos ou agentes que interagem entre si ao longo do tempo e de formas distintas. A troca de energia entre as partes e com o meio externo permite que os sistemas se auto organizem de modo que o todo se torna mais complexo.

Como os sistemas não são lineares, causa e efeito não assumem uma relação proporcional e também não é possível prever precisamente quando um comportamento aleatório vai ocorrer (FRANCO, 2013).

Cabe ressaltar que, no sistema complexo que a autonomia representa, há fatores cruciais inter-relacionados, como, por exemplo, o aprendiz, o professor, a instituição, o material, os contextos social e político, a legislação e a tecnologia. Como a autonomia não é exclusivamente inata, um ou mais desses fatores podem incentivar ou reprimir o desenvolvimento da autonomia (PAIVA, 2006).

Em se tratando da relação entre tecnologia e autonomia, mais especificamente o uso da internet na aprendizagem de língua estrangeira, Warschauer afirma que o conceito de autonomia deve ir além de sua aplicação no auto direcionamento no uso da língua e na tecnologia atual. Para ele, o termo deve ser aplicado "à capacidade de desenvolver, explorar, avaliar e adaptar a nova tecnologia à medida que evolui” (2002, p. 457).

Paiva alega que o material da web pode propiciar maior autonomia ao aluno, uma vez que não é possível prever todas as conexões estabelecidas pelas inúmeras possibilidades que o hipertexto digital oferece. Segundo a autora, o ambiente on-line, “além de ser mais propício a um tipo de educação menos conservadora, representa um estímulo a abordagens de ensino mais centradas no aluno" (PAIVA, 2001, p. 97).

Essa ênfase no aprendiz por meio da aprendizagem socialmente mediada está diretamente adjunta à perspectiva sociocultural de autonomia. Segundo Oxford, podemos dividir a perspectiva sociocultural (também conhecida como sócio interacionista) em I e II.

\footnotetext{
Na perspectiva sociocultural I, a autonomia é autorregulada, adquirida por meio de interação social com um mediador mais competente, podendo a mediação ocorrer por parte de outro aprendiz, professor, livros ou até mesmo a tecnologia.

Na perspectiva sociocultural II, entretanto, “o aprendiz não é tão importante quanto a participação na comunidade; e essa perspectiva enfatiza o contexto da autonomia em detrimento de o indivíduo exercê-la. (OXFORD, 2003, p. 87, tradução nossa)
}

A perspectiva sociocultural (I e II) nos ajuda a estabelecer um paralelo entre autonomia e tecnologia e, consequentemente, justifica a escolha dos recursos e ferramentas digitais, 
apresentados na seção seguinte, para a promoção da autonomia do professor e do aluno. A aprendizagem autônoma em contextos mediados por novas tecnologias pode ocorrer individualmente ou colaborativamente, isto é, os sujeitos podem controlar sua própria aprendizagem ou esta pode ocorrer por meio da participação ativa em uma comunidade específica, como um grupo de alunos no laboratório de informática ou um grupo de professores em um fórum de discussão on-line.

Os pesquisadores Haythornthwaite, Kazmer, Robins e Shoemaker (2000) apontam para uma tendência da educação on-line - conhecida como dupla aprendizagem, da disciplina e da tecnologia - em que, além de aprender a usar uma nova tecnologia, os alunos aprendem a interagir a distância. Em outras palavras, à medida que o aluno virtual passa a dominar a tecnologia utilizada na educação on-line, tornando-se mais autônomo em relação à aprendizagem, ele constrói seu conhecimento tanto sobre o conteúdo desejado quanto sobre a própria tecnologia. A dupla imersão de conhecimentos geralmente começa com a familiarização com as ferramentas necessárias para a realização de atividades sobre o conteúdo-alvo. Bruce e Hogan (1999) acreditam que, ao longo do curso, a tecnologia passa a ser transparente para o aluno e deixa, finalmente, de ser o foco da aprendizagem.

\section{Recursos digitais: possibilidades para o desenvolvimento da autonomia}

Encontramos uma série de recursos digitais disponíveis gratuitamente na internet, como websites e ferramentas digitais, que podem ser utilizados por professores e alunos. Ao considerarmos a língua inglesa como o idioma mais utilizado na internet, destacam-se as inúmeras possibilidades do uso de recursos digitais para o contexto de ensino-aprendizagem de inglês. Para o docente, como veremos a seguir, é possível elaborar suas próprias atividades pedagógicas e compartilhá-las com colegas da área. Para o aprendiz, é possível, por exemplo, fazer atividades com feedback automático, consumir e produzir conteúdos em texto, áudio ou vídeo, além de poder interagir em inglês com pessoas de diferentes nacionalidades,

\section{Recursos digitais para o professor}

Como já vimos na introdução deste artigo, a internet é uma valiosa fonte de informação e de comunicação entre as pessoas. Para o professor, os websites voltados para o ensino de inglês representam fontes de textos atuais e de temas diversificados. Em alguns desses sites, os docentes têm a possibilidade de participar de comunidades de prática pedagógica e de trocar mensagens entre colegas de profissão. 
Uma maneira simples de pesquisar trabalhos acadêmicos, como artigos científicos, capítulos de livros, teses, resumos, é por meio da rede social Research Gate (https://www. researchgate.net) ${ }^{2}$, que possui mais de 20 milhões de usuários. Criada em 2008, trata-se de uma rede social livre que conecta pesquisadores e cientistas, o que ajuda a criar visibilidade para a produção científica nacional e internacional de todas as áreas do conhecimento.

Os portais Teaching English (https://www.teachingenglish.org.uk) e Using English for Academic Purposes (https://www.uefap.co.uk) oferecem uma vasta gama de recursos voltados para o ensino de inglês.

O Teaching English é elaborado pelo Conselho Britânico e disponibiliza sugestões de atividades, planos de aula, fóruns de discussão, detalhes sobre conferências profissionais e artigos acadêmicos, entre outras informações relevantes para o profissional da área de Letras. O Using English for Academic Purposes, por sua vez, apresenta uma grande variedade de conteúdos práticos e teóricos para quem precisa usar a língua inglesa com propósitos acadêmicos. O portal é útil não somente para o professor de língua inglesa, que procura informações sobre leitura e redação de textos acadêmicos (resumos, palestras, etc.), como também para o aprendiz que precisa de dicas (cf. Figura 1), orientação de estudos e exercícios com respostas em inglês para fins acadêmicos.

Figura 1 - Página com estratégias para a leitura de textos acadêmicos, apresentadas no site Using English for Academic Purposes

\begin{tabular}{|c|c|}
\hline Fither & a \\
\hline Home & \\
\hline Accuracy & $>$ \\
\hline Grammar in EAP & $>$ \\
\hline Listening & $>$ \\
\hline Materials & $>$ \\
\hline Preparing & $>$ \\
\hline Reading & $>$ \\
\hline Speaking & $>$ \\
\hline Vocabulary & $>$ \\
\hline Writing & $>$ \\
\hline About UEIAP & $>$ \\
\hline EAP in General & $>$ \\
\hline
\end{tabular}

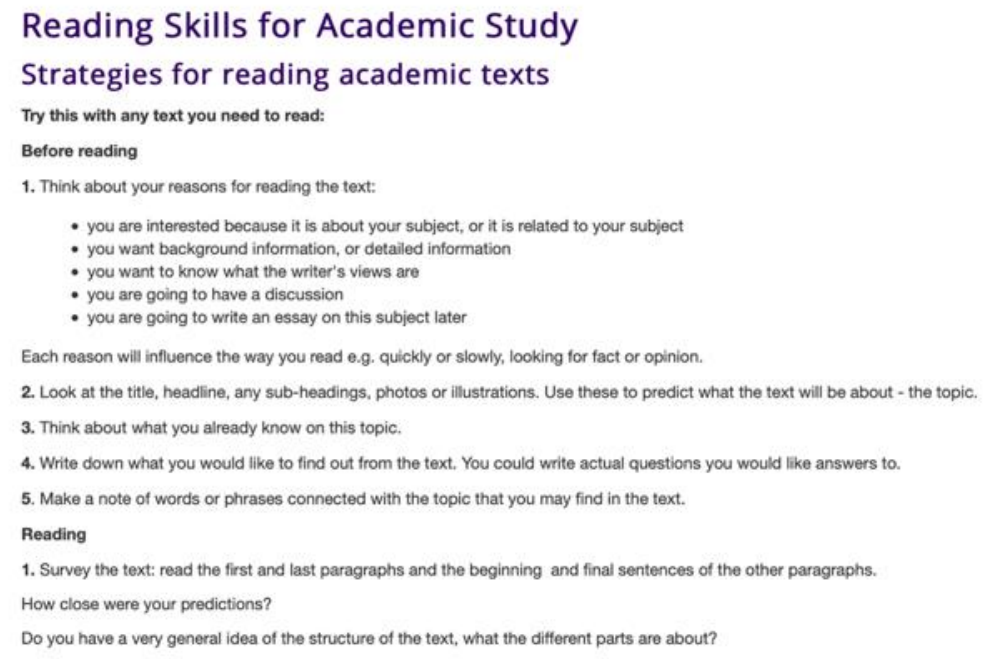

Fonte: capturado pelo autor

\footnotetext{
2 Todos os links indicados neste artigo foram acessados em 18-12-2020.
} 
Para o professor que não dispõe de tanto tempo para preparar seu próprio material didático, existem sites com atividades pedagógicas prontas para serem utilizadas na sala de aula presencial ou virtual. Em English Language Teaching Resources (https://www.englishwsheets.com), por exemplo, são disponibilizadas centenas de folhas com exercícios sobre aspectos linguísticos (gramática e vocabulário) para estudantes de inglês em vários estágios de aprendizagem.

Em se tratando de ferramentas digitais para a produção de materiais e atividades didáticas, destacam-se os sistemas ou programas de autoria. Um conhecido sistema de autoria é o Hot Potatoes (https://hotpot.uvic.ca) ${ }^{3}$. Ele oferece seis tipos de exercícios interativos em formato de página simples da web (HTML): múltipla escolha, perguntas curtas, palavrascruzadas, ordenação de frases, "correlacione..." e "preencha as lacunas". As atividades podem ser realizadas tanto on-line quanto off-line e oferecem feedback imediato.

Outro exemplo de sistema de autoria é o ELO - Ensino de Línguas Online (https://elo.pro.br/cloud/), desenvolvido pelo Prof. Dr. Vilson Leffa (UCPel). Ao acessar o referido website, é possível encontrar um amplo repositório de atividades, que incluem reconstrução de texto, lacunamento (cloze, em Inglês), sequência textual, jogo da memória, múltipla escolha e exposição dialogada. Esse programa propicia, ao mesmo tempo, certa autonomia ao professor de línguas, fornecendo-lhe subsídios para que produza seu próprio material didático, e contribui para a autonomia do aluno, pois as atividades oferecem feedback imediato (cf. Figura 2) e a possibilidade de realizar exercícios de leitura de textos com dicionário acoplado.

Um exemplo de programa de autoria voltado para a produção de palavras cruzadas é o Eclipse Crossword (https://www.eclipsecrossword.com).

As atividades digitais com resposta automática produzidas com os sistemas ou programas de autoria supracitados parecem atender ao princípio de tentativa e erro, apontado por Holec (1980) em estudo sobre autonomia, conferindo maior independência para o aluno. Para esse autor, a descoberta, com ou sem a ajuda de outros parceiros mais competentes, é a metodologia básica para o treinamento do aprendiz: "ao proceder amplamente por tentativa e erro, ele [o aprendiz] pratica progressivamente" (1980, p. 42).

${ }^{3} \mathrm{O}$ software é gratuito para fins educacionais, mas é necessário fazer um registro no site do programa. 
Figura 2 - Atividade de leitura em inglês com feedback imediato, elaborada com a ferramenta ELO

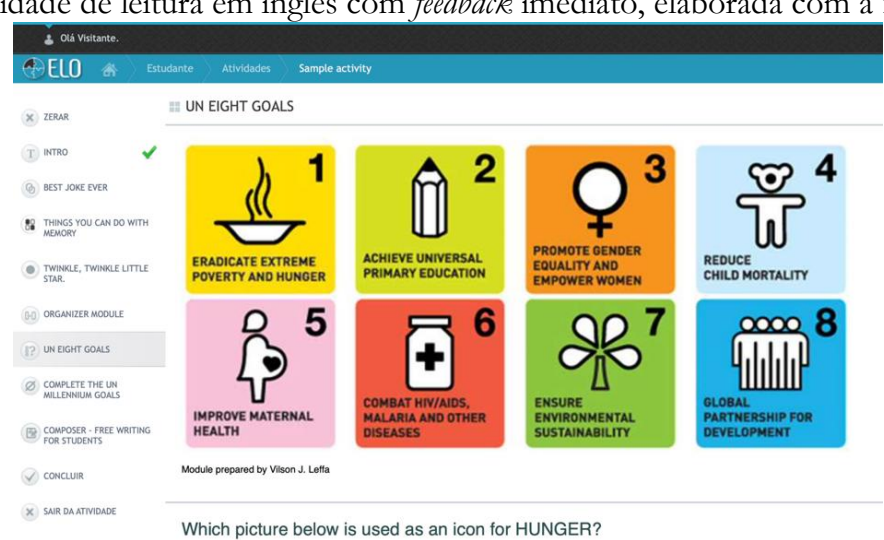

Which picture below is used as an icon for HUNGER?

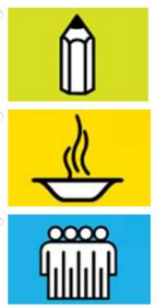

()

Fonte: capturado pelo autor

\section{Recursos digitais para o aluno}

Alguns estudos sugerem que os aprendizes envolvidos com educação a distância desenvolvem, ao longo do tempo, a habilidade de aprender de maneira autônoma. Em pesquisa sobre esse tema, Anderson (2003) reportou que alunos de cursos on-line perceberam que a internet facilitou o desenvolvimento de sua autonomia.

Dois sites que disponibilizam o serviço de criação de vídeos são o Canva (https://www.canva.com) e o Animaker (https://www.animaker.co). Por meio deles, os alunos podem trabalhar diferentes gêneros orais, como propagandas, entrevistas, discursos. Além disso, os vídeos produzidos podem ser compartilhados em sites como YouTube (https://www.youtube.com) ou Vimeo (https://vimeo.com).

O desenvolvimento da autonomia do aluno por meio da criação e compartilhamento de vídeos na internet tem como fundamentação teórica os trabalhos de Holec sobre a necessidade de ter como foco a perspectiva do aprendiz em relação aos objetivos e processos de aprendizagem. Para esse autor, equipar-se com os meios para empreender seu próprio programa de aprendizagem pressupõe que, no mínimo, você acha possível ser ao mesmo tempo "produtor" e "consumidor" de um programa desse tipo. Isso vai contra as atitudes habituais dos membros da nossa sociedade de consumo moderna; na verdade, para o indivíduo, isso 
significa retirar-se, de certa forma, uma vez que o procedimento habitual de aquisição de "bens" (neste caso, a competência em língua inglesa) não é criativo (1985, p. 182).

Em outras palavras, como a autonomia está preocupada com os interesses e necessidades dos estudantes, ser prosumer (neologismo criado pela junção dos termos, em inglês, producer e consumer, isto é, "produtor" e "consumidor", respectivamente) mostra-se imperativo para a aprendizagem de inglês em um mundo globalizado. Assim, o sistema educacional, em vez de ser mais um prestador de serviços para a economia mundial (isto é, um ambiente em que as escolhas dos alunos são limitadas e assemelham-se às escolhas de um consumidor), deve potencializar a autonomia do estudante por meio de decisões que satisfaçam suas particularidades, levando-o a ser também um produtor de informações.

Outro exemplo de serviço que fomenta a autonomia do aluno prosumer é a criação de publicações on-line, como revistas, livros e manuais. Entre os mais acessados, estão:

Calaméo (https:/ / en.calameo.com);

Crello (https://crello.com);

Myebook (https://myebook.com);

My storybook (https://mystorybook.com), como mostra a Figura 3.

Figura 3 - Publicação on-line em inglês para o público infantil, elaborada com o serviço gratuito My storybook

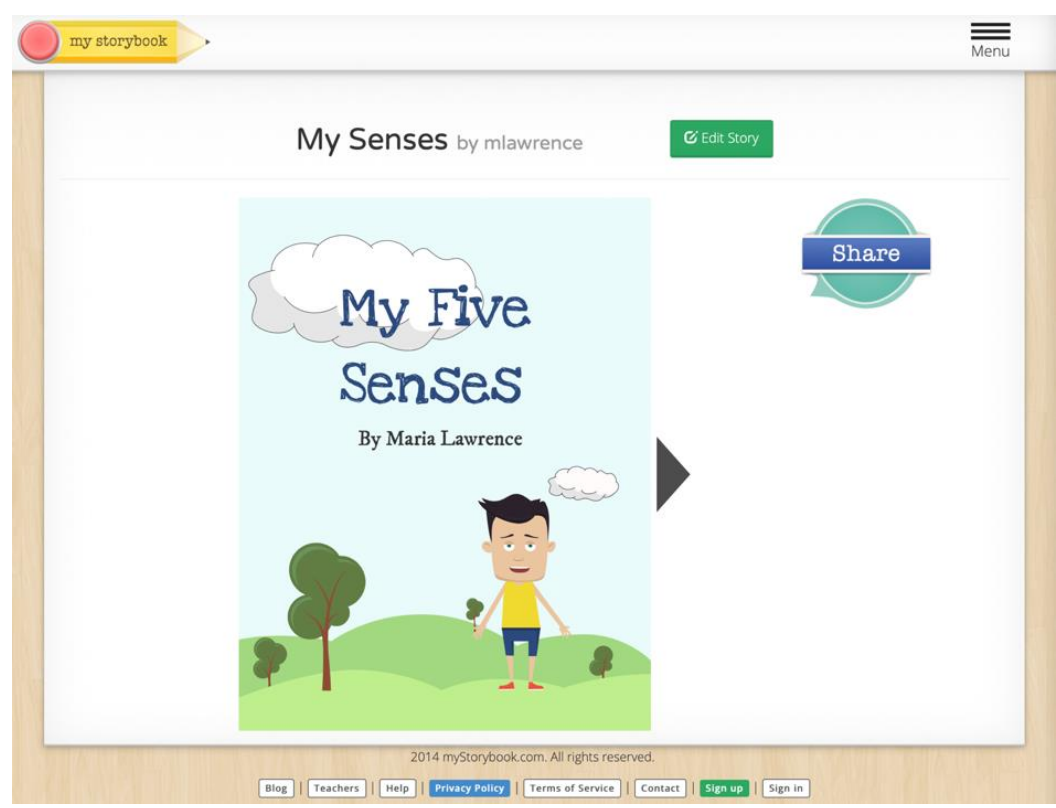

Fonte: capturado pelo autor

Ainda sobre a produção e o consumo de conteúdo multimídia com aplicação na aprendizagem de inglês, temos serviços disponíveis em Voicethread 
(https://voicethread.com/products/k12/) e Voki (https://1-www.voki.com). No primeiro, os aprendizes podem elaborar apresentações interativas com comentários de voz, vídeo ou texto; no segundo, têm a possibilidade de criar personagens virtuais por meio de mensagens de voz, gravadas por microfone, conversão de texto ou utilização de arquivo de som já existente.

Outro serviço gratuito que pode propiciar o desenvolvimento da autonomia do aprendiz de inglês encontra-se na página Witty Comics! (http://www.wittycomics.com), que permite a criação e o compartilhamento de tirinhas.

Entre os sites dedicados à aprendizagem de língua inglesa em geral, quatro se destacam:

- Activities for ESL Students (http://a4esl.org) - parte integrante do projeto The Internet TESL Journal (http://iteslj.org) -, com testes, palavras cruzadas e outros exercícios nas áreas de gramática e vocabulário, organizados por nível de dificuldade e com acesso às respostas corretas;

- BBC Skillswise (https://www.bbc.co.uk/teach/skillswise), em que os alunos podem estudar - por meio de vídeos, jogos, testes e exercícios - estratégias para o desenvolvimento das quatro habilidades (reading, writing, listening e speaking);

- World English (www.world-english.org), constantemente atualizado e com atividades e testes relacionados a tópicos de vocabulário, gramática, quatro habilidades e literatura. Há também uma área própria para troca de mensagens entre amigos virtuais (epals);

- Many Things (www.manythings.org), que traz várias atividades de leitura com áudio, quizzes e jogos sobre itens lexicais e gramaticais.

Há também sites exclusivamente destinados à habilidades de compreensão oral em inglês, conforme destacamos a seguir:

- ELLLO, English Listening Lesson Library Online (https://www.elllo.org), no qual todos os vídeos oferecem opção de legenda, e os áudios (sempre com a possibilidade de download), de transcrição. Há jogos e atividades de compreensão do tipo múltipla escolha, organizados por nível de dificuldade e tema, além de uma grande variedade de áudio de falantes de diversas nacionalidades.

- Voices of America - Learning English (https://learningenglish.voanews.com), no qual encontramos vídeos legendados e podcasts sobre notícias de todo o mundo com dois terços de sua velocidade original. Segundo os responsáveis pelo site, essa 
redução ajuda os aprendizes de inglês a acompanhar claramente cada palavra das reportagens.

Uma página da internet de grande utilidade no que diz respeito à compreensão e produção oral em inglês é o EnglishCentral (https://englishcentral.com). Nela, o aprendiz pode aprimorar o idioma de maneira autônoma por meio de vídeos autênticos, organizados por nível de dificuldade, com ou sem legendas, e praticar a fala usando o microfone do computador. Por causa da tecnologia de reconhecimento de voz, a pronúncia do usuário é avaliada e uma pontuação é atribuída com base no desempenho em cada etapa cumprida. O EnglishCentral dispõe de um catálogo com mais de cinquenta tópicos como esportes, filmes, meio ambiente, música, notícias, política, saúde, etc.

A página Sounds of English (https://www.soundsofenglish.org) trabalha dois importantes aspectos que envolvem a compreensão e a produção oral: pronúncia e entonação. Além de apresentar sons específicos de pares mínimos e word stress em inglês, o Sounds of English oferece aos professores dicas importantes e exercícios de pronúncia para impressão e uso em sala de aula.

Encontramos ainda sites voltados tanto para o ensino quanto para a aprendizagem de Inglês como língua estrangeira ou segunda língua. Destacam-se os seguintes:

- EnglishClub.com (https://www.englishclub.com), site com conteúdo extenso e grande variedade de links para explicações e atividades, planos de aula e informações sobre a prática do ensino da língua inglesa destinadas aos profissionais da área.

- English as Second Language for Teachers and Students (https://www.thoughtco.com/esl-4133095), em que há um grande número de links para artigos sobre diversos temas, como pronúncia, planos de aula, gramática, vocabulário, ensino de inglês, inglês para negócios, testes, entre outros.

- Sobre o ensino de gramática, professores e alunos encontram resumos de vários tópicos da língua inglesa e atividades nos seguintes sites:

- Daily Grammar (http://www.dailygrammar.com/archive.html), cujos exercícios estão disponíveis para impressão e vêm com chave de resposta.

- Learn English - British Council (https://learnenglish.britishcouncil.org/grammar), em que oferece prática da língua inglesa por meio de jogos, exercícios de vocabulário, gramática e atividades de compreensão escrita e oral. O portal do Conselho Britânico (British Counci) oferece conteúdo específico tanto para professores quanto para alunos de inglês. 
Uma fonte importante de textos autênticos são os portais que dão acesso a jornais de todo o mundo. Neles, o professor de idiomas encontra um rico material para elaborar suas aulas e os alunos têm a oportunidade de pesquisar e de desenvolver a prática extensiva de leitura de temas atuais. Aqui, destacamos:

- Online Newspapers (http://www.onlinenewspapers.com/index.shtml), que oferece a versão on-line de jornais famosos de diversos países.

\section{Considerações finais}

Neste artigo, vimos que autonomia é um termo complexo - as diversas definições não são excludentes, mas se complementam - e que o ciberespaço pode oferecer inúmeras oportunidades para que professores e alunos desenvolvam graus mais elevados de autonomia. Contudo, acreditamos que, para que isso, de fato, ocorra, docentes e estudantes devem ter consciência das reais necessidades e dos desejos de todos os envolvidos no processo de ensino-aprendizagem.

Ao longo deste artigo, apresentamos ferramentas e serviços disponíveis na rede (como páginas da internet e ferramentas de criação de atividades pedagógicas) que podem propiciar a autonomia de professores e alunos no contexto de ensino-aprendizagem de inglês. Precisamos, acima de tudo, refletir sobre as potencialidades da tecnologia e sua relação com a autonomia. Acreditamos que docentes e discentes, ao aprender a utilizar determinado recurso digital, adquirem empoderamento e emancipação, dois elementos importantes para o desenvolvimento de uma atitude mais autônoma em relação ao processo de ensino-aprendizagem. De acordo com Paiva (2006), as ferramentas da internet podem aumentar as oportunidades de aprendizagem, emancipando os alunos em seu caminho rumo à autonomia. Benson (1987), além de reconhecer o potencial da tecnologia no desenvolvimento da autonomia, acredita que esta é necessária para que o indivíduo possa usufruir de tudo o que os ambientes virtuais oferecem.

\section{AUTONOMY AND THE USE OF DIGITAL RESOURCES IN ENGLISH LANGUAGE TEACHING AND LEARNING}

\footnotetext{
ABSTRACT: The current article, organized into two parts, aims at offering both theoretical and hands-on knowledge by focusing on the use of digital resources in the development of the autonomy of the agents involved in the English teaching-learning process. In the first part, using a theoretical approach guided by the complexity theory, we propose to offer a brief overview of the concept of autonomy as a complex adaptive system. In the second part, using a hands-on approach, we seek to discuss possible uses of various digital resources, available for free on the internet, that can positively contribute to the promotion of the teacher and learner autonomy.
} 
KEYWORDS: digital resources; English language teaching; autonomy; complexity theory

\section{REFERÊNCIAS}

ANDERSON, Terry. Modes of interaction in distance education: recent developments and research questions. In: MOORE, Michael Grahame; ANDERSON, William G. (Orgs.) Handbook of distance education. Lawerence Enbawm Associates, 2003.

BENSON, Phil. The philosophy and politics of learner autonomy. In: BENSON, Phil; DICKINSON, Leslie. Self-instruction in language learning. Cambridge: Cambridge University Press, 1987.

BRUCE, Bertram C.; HOGAN, Maureen P. The disappearance of technology: toward and ecological model of literacy. In: REINKING, David; MCKENNA, Michael C.; LABBO, Linda D.; KIEFFER, Ronald D. (Orgs.) Handbook of literacy and technology: transformations in a post-typographical world. Hillsdale: Erlbaum, 1999.

DICKINSON, Leslie. Self-instruction in language learning. Cambridge: Cambridge University Press, 1991.

FRANCO, Claudio de Paiva. Autonomia na aprendiragem de inglês: um estudo com nativos digitais sob as lentes do caos e da complexidade. 2013. 201f. Tese (Doutorado em Estudos Linguísticos) Faculdade de Letras, Universidade Federal de Minas Gerais. Belo Horizonte, 2013. Disponível em: http://claudiofranco.com.br/media/tese-claudiofranco.pdf. Acesso em: 18 dez. 2020.

FREIRE, Paulo. Pedagogia da autonomia - saberes necessários à prática educativa. 35. ed. Rio de Janeiro: Paz e Terra, 2007.

HAYTHORNTHWAITE, Caroline; Community development among distance learners: temporal and technological dimensions. Journal of Computer-Mediated Communication, v. 6, i. 1, 2000. Disponível em: https://doi.org/10.1111/j.1083-6101.2000.tb00114.x Acesso em: 18 dez. 2020.

HOLEC, Henri. Autonomy and foreign language learning. Oxford: Pergamon, 1981.

HOLEC, Henri. Learner training: meeting needs in self-directed learning. In: ALTMAN, H. B.; JAMES, C. V. (Orgs.) Foreign language learning: meeting individual needs. Oxford: Pergamon, 1980.

HOLEC, Henri. On autonomy: some elementary concepts. In: RILEY, P. (Org.) Discouse and learning. London: Longman, 1985.

LARSEN-FREEMAN, Diane. Chaos/complexity science and second language acquisition. Applied Linguistics, Oxford, v. 18, n. 2, 141-165, 1997.

LITTLE, David. Learner autonomy: definitions, issues and problems 1. Dublin: Authentic, 1991.

OXFORD, Rebecca L. Toward a more systematic model of L2 learner autonomy. In: PALFREYMAN, David; SMITH, Rirchard C. (Orgs.) Learner autonomy across cultures: language 
education perspectives. Basingstoke: Palgrave Macmillan, 2003. Disponível em: https://doi.org/10.1057/9780230504684_5. Acesso em: 18 dez. 2020.

PAIVA, Vera Lúcia Menezes de Oliveira e. Autonomia e complexidade. Linguagem e Ensino, Pelotas, v. 9, n. 1, 77-127, 2006. Disponível em: https://periodicos.ufpel.edu.br/ojs2/index.php/rle/article/view/15628. Acesso em: 18 dez. 2020.

PAIVA, Vera Lúcia Menezes de Oliveira e. A www e o ensino de Inglês. Revista Brasileira de Linguística Aplicada, Belo Horizonte, v. 1, n. 1, 93-116, 2001. Disponível em:

http://www.veramenezes.com/www.htm. Acesso em: 18 dez. 2020.

PENNYCOOK, Alastair. Cultural alternatives and autonomy. In: BENSON, Phil: VOLLER, Peter (Orgs.) Autonomy and independence in language learning. New York: Addison Wesley Longman, 1997.

ROGERS, C. R. Freedom to learn. Colombus: Charles E. Merril, 1969.

TAVARES, Kátia Cristina do Amaral; FRANCO, Claudio de Paiva. Ensino de leitura em língua portuguesa: de concepções teóricas a propostas de atividades didáticas. In: MOLLICA, Maria Cecília (Org.) Linguagem em Contextos. Rio de Janeiro: Editora 7Letras, 64-77, 2014.

VOLLER, Peter (Org.) Autonomy and independence in language learning. New York: Addison Wesley Longman, 1997.

WARSCHAUER, Mark. A developmental perspective on technology in language education. TESOL Quarterly, v. 36, n. 3, p. 453-475, 2002. Disponível em: http:/ / education.uci.edu/uploads/7/2/7/6/72769947/developmental1.pdf. Acesso em: 18 dez. 2020.

Recebido em: 27/03/2021.

Aprovado em: 06/07/2021. 